Time-Resolved Imaging of Material Response Following Laser-Induced Breakdown in the Bulk and Surface of Fused Silica

R. N. Raman, R. A. Negres, P. DeMange, S. G. Demos

February 8, 2010

Photonics West: High Energy/Average Power Lasers and Intense Beam Applications V San Francisco, CA, United States January 23, 2010 through January 28, 2010 
This document was prepared as an account of work sponsored by an agency of the United States government. Neither the United States government nor Lawrence Livermore National Security, LLC, nor any of their employees makes any warranty, expressed or implied, or assumes any legal liability or responsibility for the accuracy, completeness, or usefulness of any information, apparatus, product, or process disclosed, or represents that its use would not infringe privately owned rights. Reference herein to any specific commercial product, process, or service by trade name, trademark, manufacturer, or otherwise does not necessarily constitute or imply its endorsement, recommendation, or favoring by the United States government or Lawrence Livermore National Security, LLC. The views and opinions of authors expressed herein do not necessarily state or reflect those of the United States government or Lawrence Livermore National Security, LLC, and shall not be used for advertising or product endorsement purposes. 


\title{
Time-resolved imaging of material response following laser-induced breakdown in the bulk and surface of fused silica
}

\author{
Rajesh N. Raman, Raluca A. Negres, Paul DeMange, and Stavros G. Demos \\ Lawrence Livermore National Laboratory, 7000 East Avenue, Livermore, CA, USA 94551
}

\begin{abstract}
Optical components within high energy laser systems are susceptible to laser-induced material modification when the breakdown threshold is exceeded or damage is initiated by pre-existing impurities or defects. These modifications are the result of exposure to extreme conditions involving the generation of high temperatures and pressures and occur on a volumetric scale of the order of a few cubic microns. The response of the material following localized energy deposition, including the timeline of events and the individual processes involved during this timeline, is still largely unknown.

In this work, we investigate the events taking place during the entire timeline in both bulk and surface damage in fused silica using a set of time-resolved microscopy systems. These microscope systems offer up to 1 micron spatial resolution when imaging static or dynamic effects, allowing for imaging of the entire process with adequate temporal and spatial resolution. These systems incorporate various pump-probe geometries designed to optimize the sensitivity for detecting individual aspects of the process such as the propagation of shock waves, near-surface material motion, the speed of ejecta, and material transformations. The experimental results indicate that the material response can be separated into distinct phases, some terminating within a few tens of nanoseconds but some extending up to about 100 microseconds. Overall the results demonstrate that the final characteristics of the modified region depend on the material response to the energy deposition and not on the laser parameters.
\end{abstract}

Keywords: Time-resolved imaging, microscopy, fused silica, damage initiation, surface damage, bulk damage

\section{INTRODUCTION}

Laser-induced breakdown (LIB) limits the performance of optical materials employed in high average power laser applications. Industrial applications concerned with damage of optical components within these laser systems include microlithography, micromachining, and laser welding. In addition, laser systems designed to achieve laser-driven inertial confinement fusion operate near the LIB threshold of their component optics. LIB, which has shown to lead to modification of the electronic and structural properties of the host material [1-7], can eventually lead to the formation of a damage site. Post-mortem characterization of laser-induced damage is widely used and employs various imaging tools, for example scanning and transmission electron microscopies [5], white light scattering and photoluminescence microscopies [1,2], and optical coherence tomography [1] to name a few. These techniques are very useful but do not provide a complete timeline of the processes occurring during laser-induced damage. For example, in both surface and bulk damage, a network of cracks surrounding the damage site reveals mechanical damage and suggests transient high temperature and pressure in the material [4-5]. Indeed, extreme temperatures have been measured during energy deposition of a damage event [8], though with limited temporal resolution. Also, photoluminescence provides evidence of material modifications on the molecular level, where host material stoichiometry has been disrupted and results in the generation of optically absorbing defects [2-3]. In addition, voids are observed, implying that ejection or evaporation of material (in the case of surface damage) [9] or material compaction (in the case of bulk damage) [4,10] had occurred at some time during the event. Only a limited number of studies of the dynamic processes during a damage event have been performed [8-9,11]. Thus, the sequence of material modifications along the damage timeline leading to the final state of the damage site has not been adequately documented. Among the processes not well understood include the nature of the initial material response, the means by which energy is transported in the material once deposited, and the resulting material displacement and lattice transformation.

The objective of this paper is to demonstrate the capabilities of a time-resolved microscope system with adequate spatial and temporal resolution to image the dynamic processes occurring during confined laser energy deposition in the bulk and on the surface of fused silica optical components. This system consists of various configurations, with capabilities 
such as multi-view imaging and imaging of multiple time points within the same damage event, each optimized to capture specific aspects of the damage timeline.

\section{EXPERIMENTAL SETUP: IMAGING BULK DAMAGE INITIATION}

\subsection{Time-resolved, polarization-sensitive transmission geometry}

We developed a time-resolved microscope system in order to capture the dynamic events during laser-induced bulk damage in optical components. Experiments were carried out on a fused silica optical window measuring $5 \mathrm{~cm}$ in diameter and $1 \mathrm{~cm}$ in thickness. A pump and probe technique was employed as follows: a focused pump beam (355-nm, 3-ns FWHM) initiates damage in the bulk of the sample via laser-induced breakdown. The damage site was illuminated in a transmission geometry using polarized strobe light from a probe pulse (532 nm, $4.5 \mathrm{~ns}$ FWHM), as illustrated in Fig. (1a). The peak-to-peak delay between the pump and probe pulses was controlled by external triggering and adjustable out to $100 \mathrm{~ms}$, to provide illumination of the damage site at various delay times covering the entire damage timeline.

The damage site was imaged with a microscope consisting of a long working-distance $5 \mathrm{X}$ magnification objective (Mitutoyo, Japan) and 5X magnification zoom lens. A polarizing beam-splitter following the imaging optics decomposed the image into two components with polarization states perpendicular to each other (parallel and orthogonal, respectively, relative to the probe pulse polarization state). Each component was directed to a separate CCD camera (Spiricon, 1616 pixels $\times 1216$ pixels, individual pixel size of $4.4 \mu \mathrm{m}$ ) for simultaneous acquisition of two orthogonal polarization transmission images at each delay time point (Fig.1a). Parallel polarization images capture the local changes in index of refraction of the material, while the orthogonal polarization images map the stress fields in the regions surrounding the damage site (via depolarization). Each camera recorded three images per damage initiation event: an image of the pristine (pre-damage) site (initial), an image at the specified delay (transient), and a final static image. Static artifacts in both the transient and final images were removed by dividing these images each by the initial image. Spatial resolution on the order of $1 \mu \mathrm{m}$ was achieved.
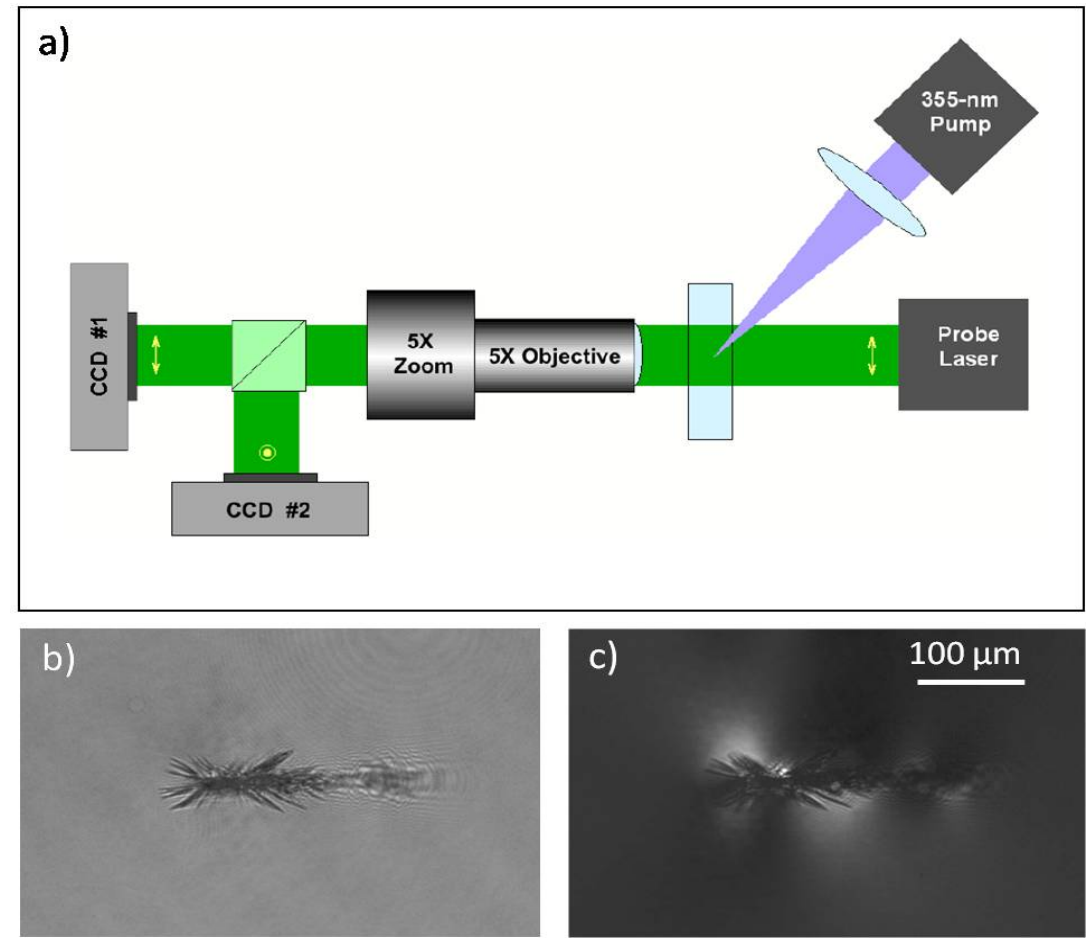

Figure 1. a) Schematic of time-resolved, polarization-sensitive microscope system for imaging processes occurring during bulk damage. b-c) Typical static (final) images of the same bulk damage event showing the b) parallel polarization component and c) orthogonal polarization component with respect to the polarization state of the probe laser. The direction of pump pulse propagation is left to right in all of the images of bulk damage. 

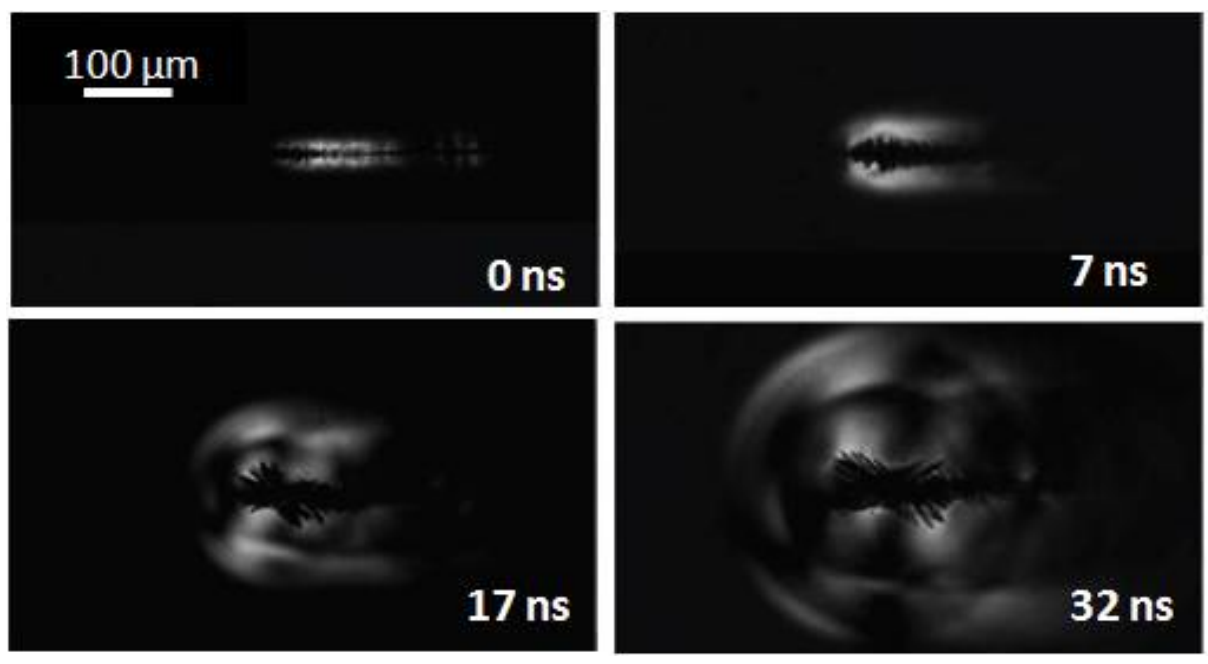

Figure 2. Orthogonal polarization images taken at various delay times capturing the processes occurring during the bulk damage timeline. Images are taken from different but similar events.

Figures $1 \mathrm{~b}$ and $1 \mathrm{c}$ show the parallel and orthogonal polarization image components of the damage site in the final state, respectively. The propagation of the pump pulse is towards the right in these images. In both images, the damage site exhibits a core region surrounded by a network of cracks. Due to the angle made by the direction of the pump beam with the probe beam, the imaging system has a depth of focus of $\sim 50 \mu \mathrm{m}$. Since we are interested in studying the dynamics of the region with core and cracks, we positioned this region within the focal plane of the imaging system, and as a result a portion of the damage site on the right-hand side of the image (which appears to be relatively free of cracks) was out of focus. Figure 1c shows the residual stress that exists in the material following a bulk damage event.

Figure 2 shows typical transient orthogonal polarization images acquired with the polarization-sensitive, transmission geometry microscope system summarizing the events observed during bulk damage. At early delay times, an elongated core region begins to form ( $0 \mathrm{~ns}$ delay) followed by the launch of a stress front. In addition, cracks are formed early on during the damage timeline and continue to grow until $\sim 30$ ns delay (by comparing crack length in the transient parallel polarization image with that in the corresponding final image). The contrast of the stress wave is higher in the orthogonal polarization image component than in the parallel image component, allowing one to clearly observe a secondary slower stress front propagating from the damage site ( $\sim 10 \mathrm{~ns}$ delay).

Time-resolved imaging of dynamic events during bulk damage allowed for the estimation of various kinetic parameters describing the material response to laser-induced damage in the bulk. In Fig. 3, the distance traveled by the initial and secondary stress fronts is plotted as a function of delay time. The estimated speed for the initial front is $\sim 5.9 \mathrm{~km} / \mathrm{s}$. The speed of the secondary stress front is slower at $\sim 3.4 \mathrm{~km} / \mathrm{s}$. By measuring the length of the cracks in the images at each delay time, one can estimate the initial crack tip growth rate after energy deposition to be $\sim 2.5 \mathrm{~km} / \mathrm{s}$ and see that crack growth rate slows down and crack growth arrests after $\sim 30$ ns.

Another observation made from the images was that the absorbing regions in the parallel polarization images at most of the delay times investigated were darker than the corresponding regions in the final state. This evidence of transient absorption can be mapped by dividing pixel-by-pixel the final image by the corresponding transient image. In this manner, regions of transient absorption appear as bright regions. A summary of the results obtained with this procedure is presented in Fig. 4. At early delays, both the core and crack regions exhibit transient absorption. Crack transient absorption terminates at $\sim 300$ ns delay while core transient absorption persists until $\sim 150 \mu$ s. Transient absorption of the probe illumination suggests that the electronic structure in the material is modified during the damage timeline. The material phase in the core region may be undergoing a phase transformation around this delay time. 


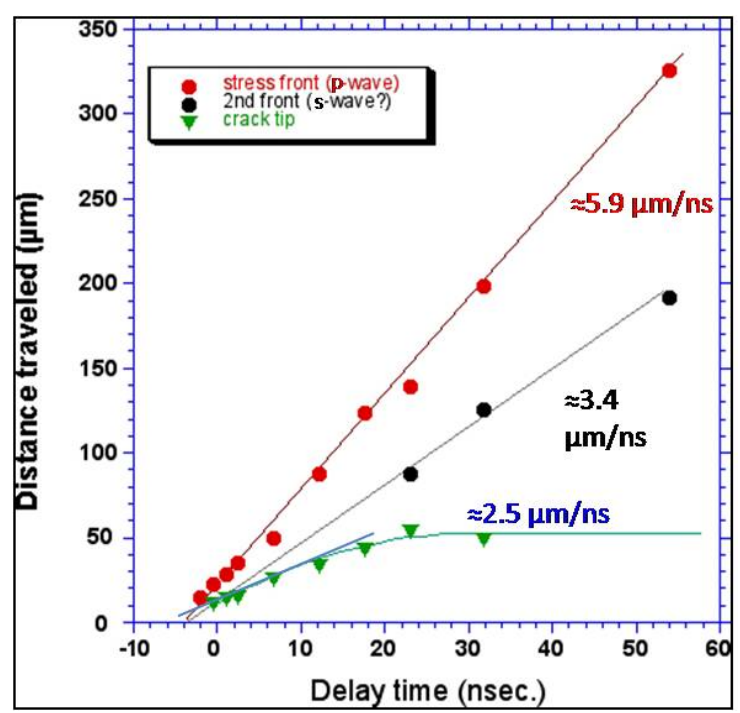

Figure 3. Estimation of kinetic parameters of various dynamic phenomena observed as a result of laser energy deposition in the bulk.
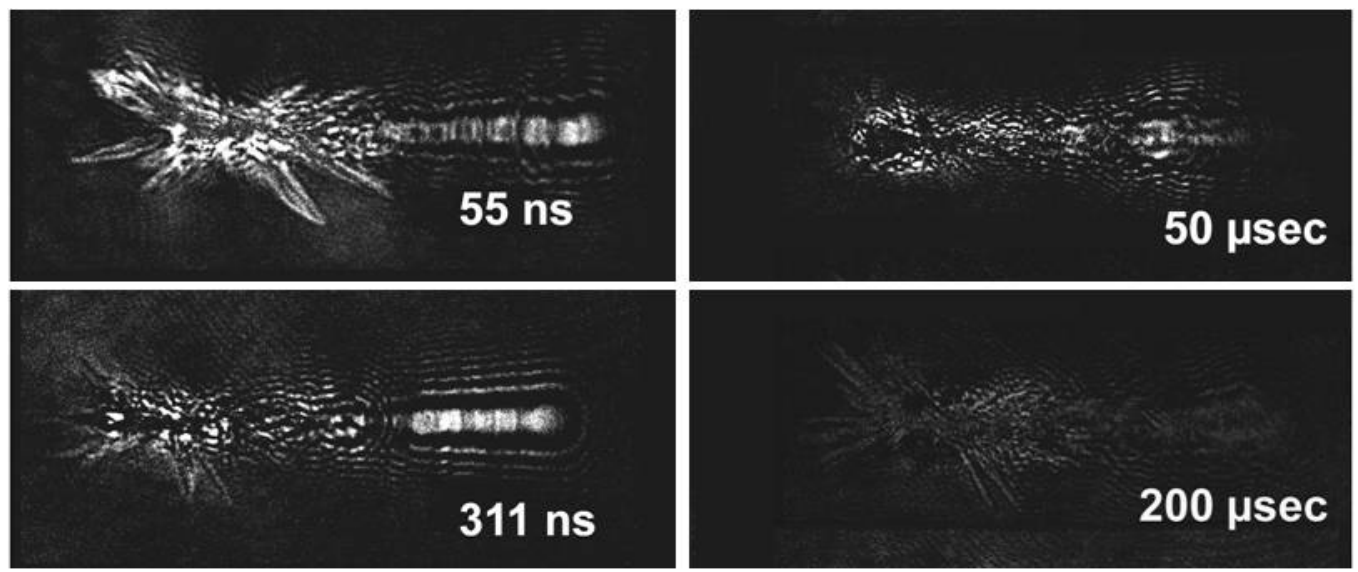

Figure 4. Ratio (final/transient) parallel polarization images highlighting regions of transient absorption (bright features).

\subsection{Back-scattering geometry}

Since there is evidence that the material is experiencing phase transformations in the core and crack regions, we hypothesized that the scattering properties of the material in this volume will also change. To test this hypothesis, we modified the previous configuration (section 2.1) by repositioning the probe beam to illuminate the damage site from the exit (camera) side of the sample and acquired images formed by scattered photons at various delay times.

The results indicate a larger back-scattering signal in the transient images relative to that of the final at certain delay times. Ratio images formed by dividing the transient image by the final image highlight regions of transient scattering (Fig. 5). Transient scattering of the core was observed to last until about $70 \mathrm{~ms}$ delay. We postulate that this region is populated with microvoids which scatter light as the liquid material in this volume contracts during cooling. 

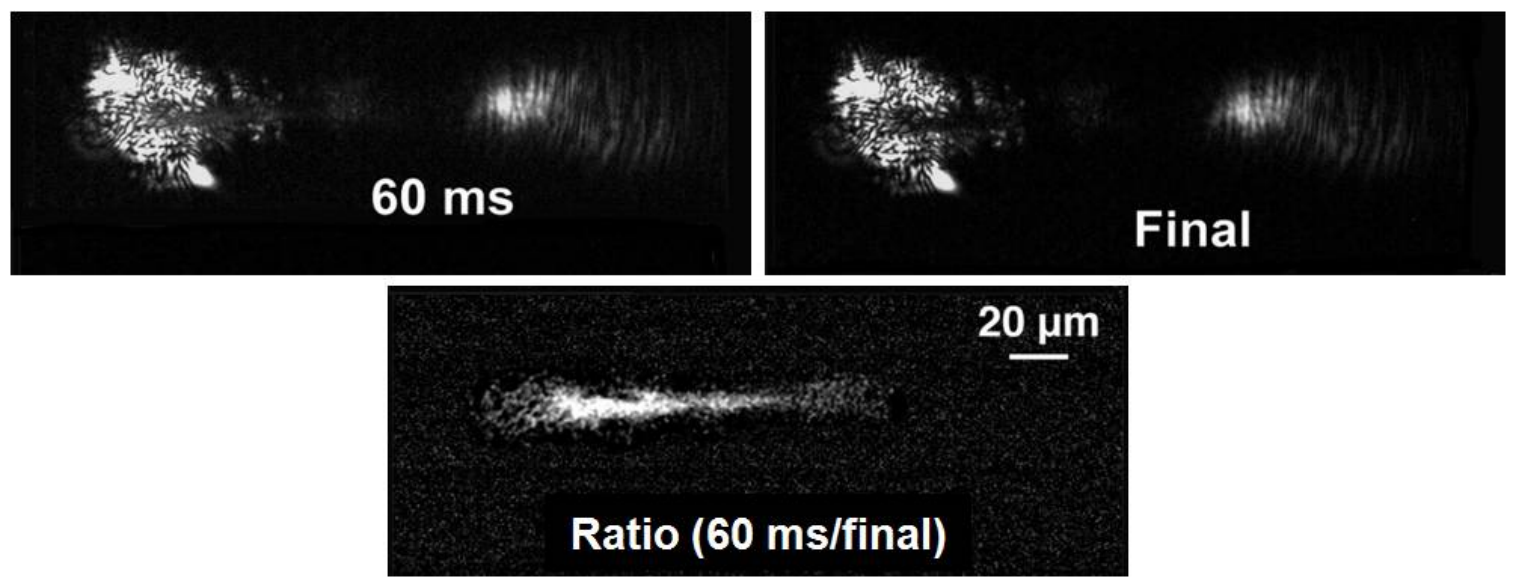

Figure 5. Images acquired using the back-scattering imaging configuration. Transient image at 60 ms delay (top left), final image (top right), and subsequent transient/final ratio (bottom).

\section{EXPERIMENTAL SETUP AND RESULTS: IMAGING EXIT SURFACE DAMAGE INITIATION}

\subsection{Basic system for simultaneous transmission and side-view imaging of surface damage}

To initiate damage on the exit surface of fused silica, the output of a pump laser operating at $355 \mathrm{~nm}$, 7-ns FWHM was focused about $2 \mathrm{~cm}$ behind the exit-surface of the sample using a 15-cm focal length lens. This arrangement provided an estimated pump beam diameter at the exit surface of $\sim 1 \mathrm{~mm}$. Pristine surface sites were first exposed to an average fluence of $\sim 30 \mathrm{~J} / \mathrm{cm}^{2}$ and damage initiation was observed with $\sim 80 \%$ probability. This procedure leads to the formation of single or multi-pit craters with average pit diameter on the order of $10-50 \mu \mathrm{m}$.

Since surface damage is associated with the formation of craters via material ejection, we initially designed our system to be able to capture this main process. In order to acquire images of crater formation and growth of its lateral dimensions as well as material ejection, two microscope systems were employed with their image planes positioned parallel and perpendicular to the sample's surface. The corresponding images are referred to as the transmission-view $(\mathrm{T}-\mathrm{V})$ and side-view (S-V) images, respectively. Both microscopes were identical, utilizing the long working-distance objective-zoom lens combination described in section 2.1, and the images were focused onto a CCD camera. The

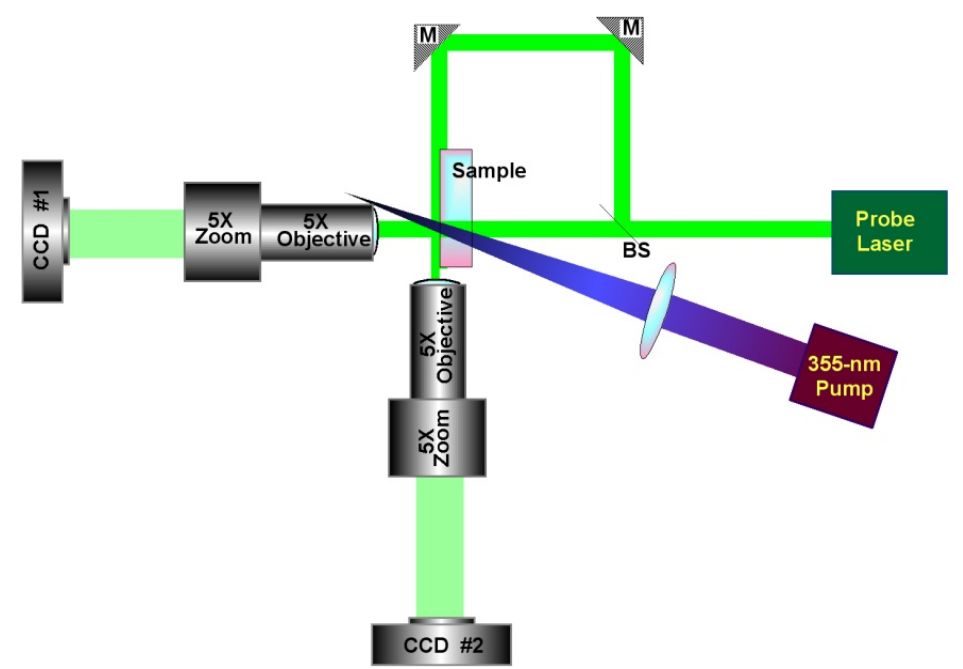

Figure 6. Basic time-resolved microscope system for simultaneous acquisition of transmission-view (T-V) and side-view (S-V) images of surface damage sites at various points during the timeline. 


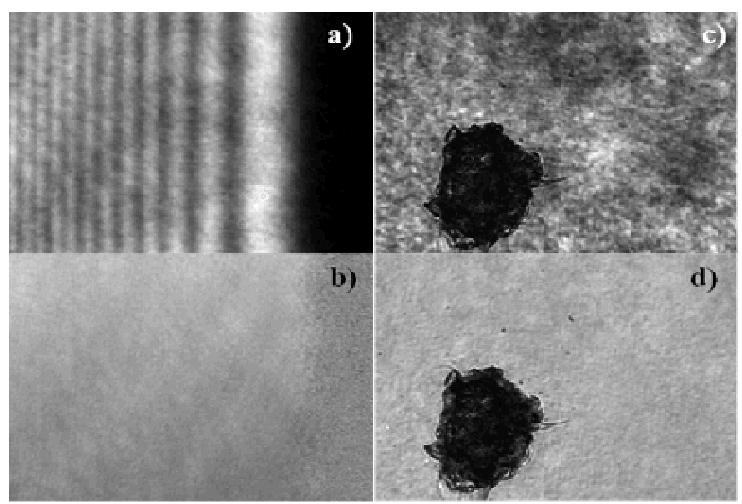

Figure 7. Demonstration of the image normalization process using pixel-by-pixel division of the raw and reference images (i.e., from damage and pristine sites, respectively). a) S-V image before and b) after normalization. c) T-V image before and d) after normalization.

resulting spatial resolution was on the order of $1 \mu \mathrm{m}$. The focal planes of the two microscopes intersected at the sample's surface at the pump beam location. In this way, the damage sites formed in this volume were imaged with optimal spatial resolution by both microscopes. A schematic of this system configuration is provided in Fig. 6 . The images were captured using strobe-light illumination provided by a $532 \mathrm{~nm}, \sim 4.5 \mathrm{~ns}$ probe pulse. Similar to the bulk imaging setup, the timing of the probe pulse output was controlled by an adjustable delay trigger in reference to the pump pulse. The pump-probe pulse jitter was determined to be $\sim 2 \mathrm{~ns}$. However, the arrival of the pump and probe pulses was separately recorded for each measurement using a set of fast photodiodes.

To remove static inhomogeneous features in the image arising from non-uniformities in the spatial profile of the probe laser beam, all images were divided by a reference image of a pristine site with no damage. Figure 7 shows examples of removal of such artifacts via image processing. In particular, Figs. 7a-b illustrate a raw transient image at 0 ns delay and the same image after normalization in the side-illumination mode. As a result, the interference fringes caused by the sample surface are largely removed. Similarly, in Figs. 7c-d the background non-uniformities in a static transmission image are likewise removed.

Typical images obtained with the basic system configuration of Fig. 6 are shown in Fig 8. Figure 8a shows an S-V image at $25 \mathrm{~ns}$ delay after exposure to the damage pulse. At least two shock fronts traveling through the air are visible which appear to originate from different points on the surface. However, no material motion is observed in this early delay time image. Figure $8 \mathrm{~b}$ displays an S-V image taken at $400 \mathrm{~ns}$ delay. A jet of particles is visible originating from the surface and traveling through the air. Particles near the surface are close together and in focus while those further downstream are more spread out and some are out of focus. The diameters of the observed particles are around 1-10 $\mu \mathrm{m}$. Also visible is the shock front traveling through the air with several particles ahead (downstream) of this front.

For estimating ejecta kinetics from the S-V images, it is necessary to precisely locate the surface of the sample, i.e. the origin of ejected particles. To locate the surface, images of the damaged surface were acquired with the S-V microscope after first blocking the S-V beam path, thus imaging the photons from the T-V beam path that were scattered by the damage site. An example is shown in Fig. 8c, where multiple scattering locations corresponding to multiple damage sites are visible as a series of vertically-oriented bright elongated spots on the right hand side of the image. Using this method, the location of the sample exit surface in the S-V images can be determined.

Figures 8d-e display images of a damage site captured by the T-V camera at two different points in time. Specifically, Fig. $8 \mathrm{~d}$ shows the transient transmission image of a damage initiation site at $7 \mathrm{~ns}$ delay, while the final crater several seconds later is shown in Fig. 8e. These images show a difference in crater dimensions, revealing that the crater is evolving over this time scale. Distinct initiation sites appear to form initially but grow to the point where some of them finally coalesce. 

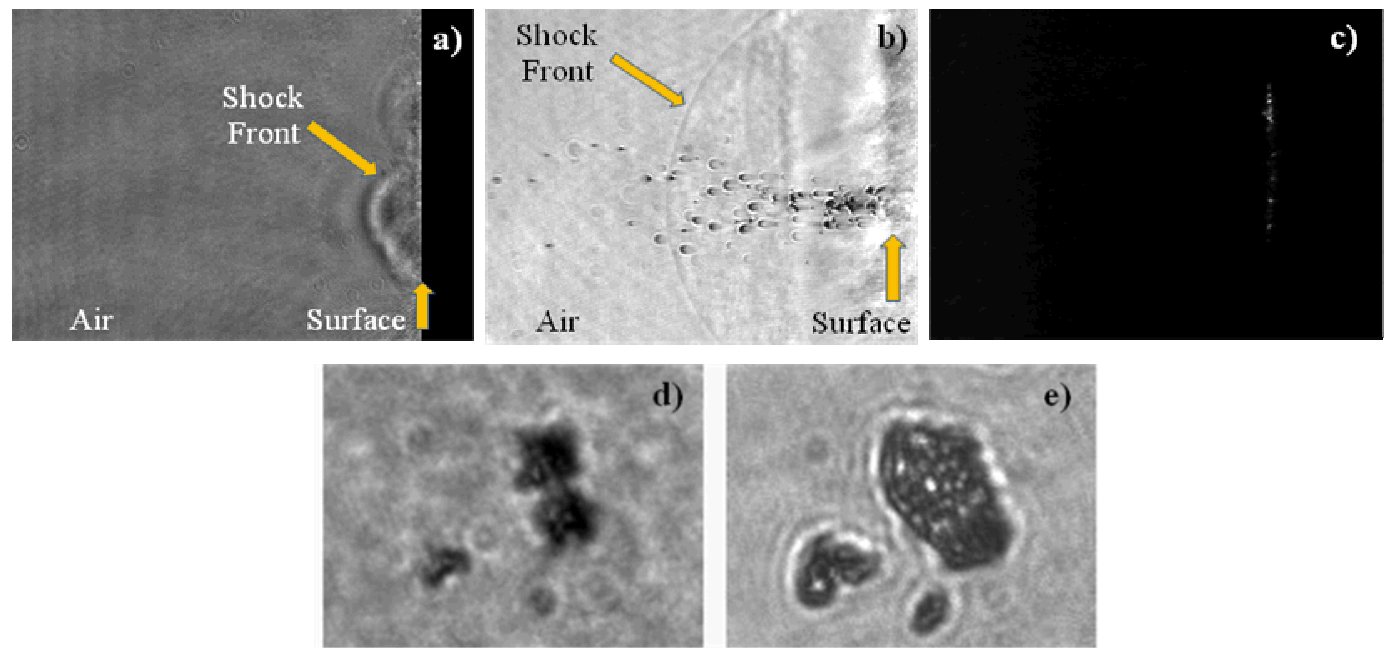

Figure 8. Typical images using simultaneous S-V and T-V imaging configurations. S-V images: a) at $25 \mathrm{~ns}$ delay, b) at 400 ns delay. c) Post-damage image captured by S-V microscope after blocking S-V beam and illuminating only with $\mathrm{T}-\mathrm{V}$ beam. Transmission images at d) transient ( $7 \mathrm{~ns}$ delay) and e) final states of the same damage site.

The basic system configuration of Fig. 6 provides simultaneous S-V and T-V shadowgraphic imaging of the same event. $\mathrm{S}-\mathrm{V}$ illumination permits the probing of a number of key processes, namely the kinetics of material cluster ejection, crater growth into the material, and near-surface phenomena prior to ejection (such as swelling and shock front generation). Material clusters appear as dark features because the probing beam is scattered and/or absorbed at these locations. This shadowgraphy mode provides a snapshot of their location and spatial characteristics during exposure to the probe pulse. The shock front in the air side is also visible because of the modulation it creates in the refractive index. With this system, average speeds of the particles and shock fronts can be estimated from the delay time of the probe pulse and distance traveled, assuming that the process started upon the damage pulse arrival (delay $=0 \mathrm{~s}$ ).

$\mathrm{T}-\mathrm{V}$ images provide views of the lateral expansion of the crater. As the absorption and scattering properties of the host material change in response to the laser energy deposition, the damage site appears as a dark object. Absorption dynamics can be measured when comparing an image in the transient state (during which the material is still undergoing modifications due to gradients in the local temperatures and pressures) to that in the final state (which represents the classical damage pit observed following a damage event). For example, the absorbing regions in Figs. $8 \mathrm{~d}$ are observed to continue to grow laterally for about $25 \mathrm{~ns}$. Information obtained via simultaneous transmission and side-view imaging makes possible the correlation between changes in lateral dimensions and with particle ejection, material swelling, and shock wave generation. Moreover, for resolving the early dynamic events as well as the ejection time of the particles, it is critical to accurately determine the location of the surface and employ the highest possible spatial resolution.

\subsection{Dark-field configuration optimized for detecting early material motion}

The results shown in Fig. 8a and 8d indicate that material changes occurring at earlier times are only partially visualized due to the obscuration in the S-V beam caused by the shock front. Thus a different configuration capable of better detecting early material motion is required.

In general, the ability of dark-field microscopy to detect small isolated particles is superior to that of bright-field microscopy. For this reason, we modified the previous configuration to employ an arrangement equivalent to dark-field microscopy. This was achieved by removing the beam-splitter and re-arranging the mirrors so as to direct the probe beam onto the exit surface location exposed to the pump pulse from the same direction used for image formation (photons detected by the S-V camera must undergo back-scattering) with the probe pulse incident at $\sim 30$ degrees with respect to the sample's exit surface. In this arrangement, transient images of damage site formation were recorded by the T-V CCD (\#1) and S-V CCD (\#2) as shown in Fig. 9. Material motion or ejection become visible by light directly scattered from the material (dark-field) rather than as a result of light obscuration (bright-field). All other laser damage and imaging parameters remained the same as in the basic system configuration shown in Fig. 6. 


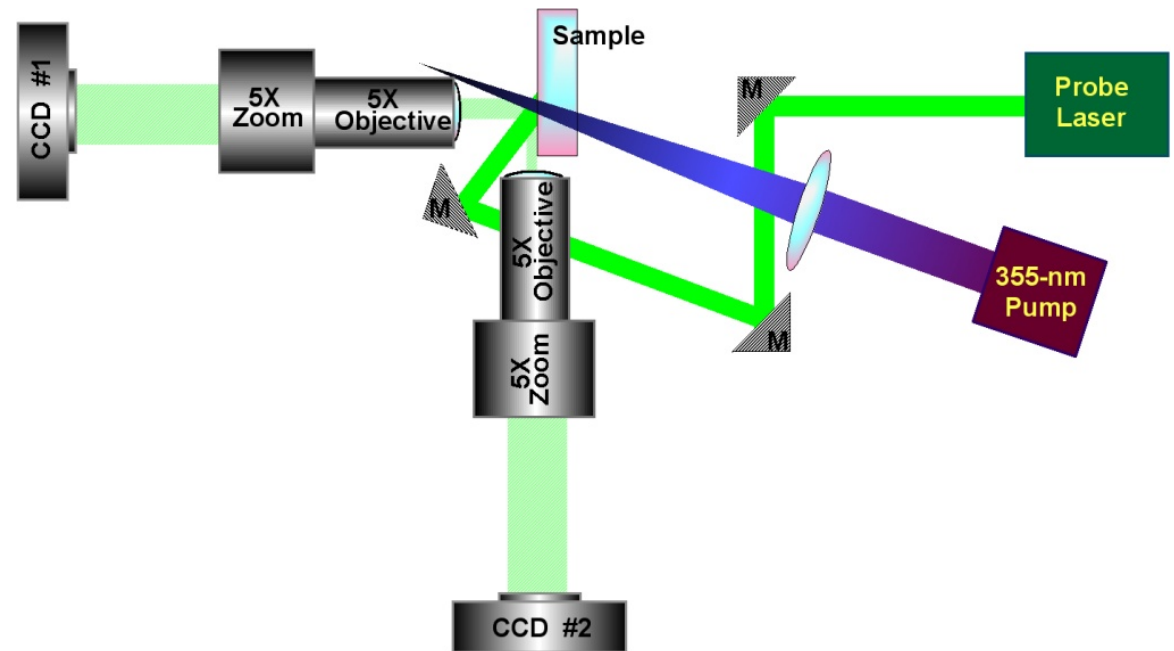

Figure 9. Pump- probe backscattering imaging configuration.

Figure 10 compares images acquired in the S-V shadowgraphy (bright-field) mode described in section 3.2 to those acquired using backscattering (dark-field) mode at the same delay times. These images are from different but similar damage events. Figures 10a-b show images acquired at 300 ns delay, where both images capture the particle ejection process. However, the advantage offered using the backscattering geometry is apparent in the images captured at early delay times. As an example, at $25 \mathrm{~ns}$ delay time, only the shock front is visible in the shadowgraphy mode (Fig. 10c), whereas material motion located behind the shock front is observable in the backscattering configuration (Fig. 10d).
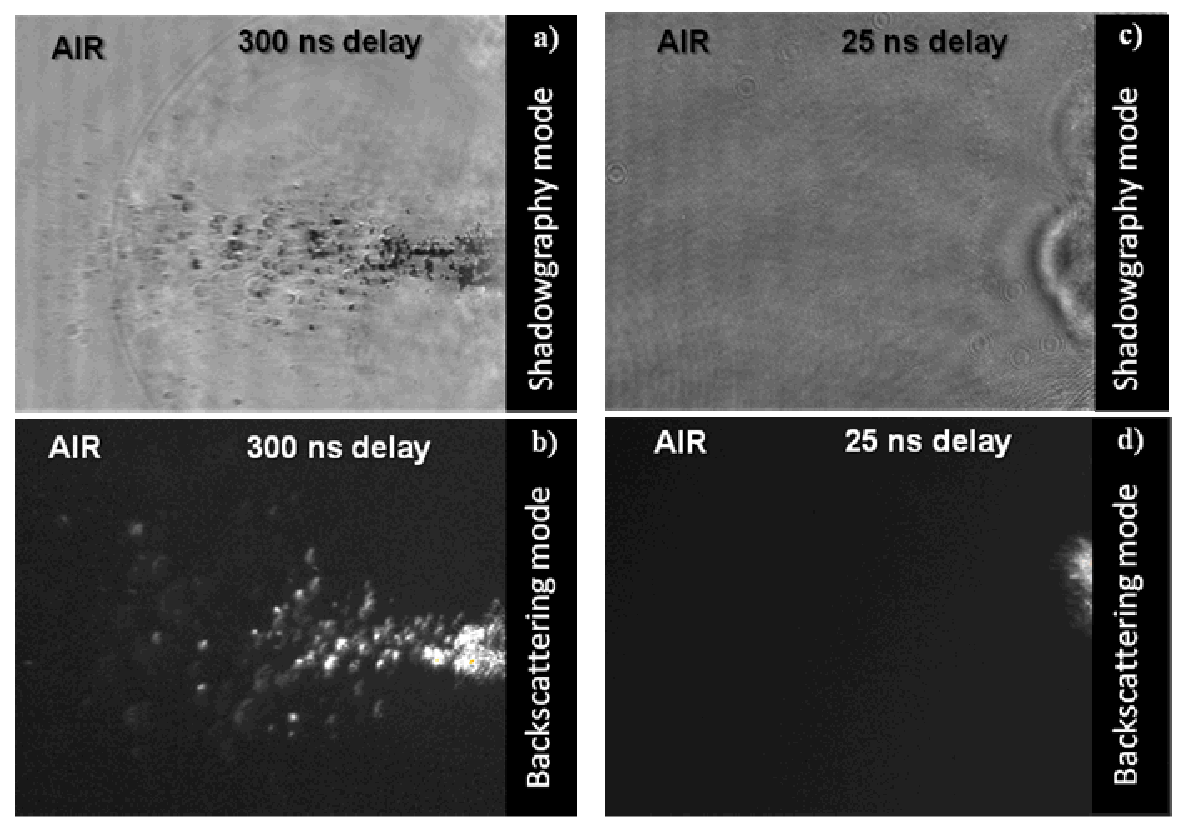

Figure 10. Images obtained by the $\mathrm{S}-\mathrm{V}$ microscope using the shadowgraphy and backscattering modes at delay times of $300 \mathrm{~ns}(\mathrm{a}-\mathrm{b})$ and $25 \mathrm{~ns}$ (c-d), respectively. Images are not from the same event and are intended to enable comparison of similar events using these different imaging modes. 
The images suggest that a swelling of the surface precedes the ejection of individual particles capturing a critical part of the early material response to localized energy deposition.

Although shadowgraphic imaging can provide a wealth of information, the backscattering imaging configuration provides better sensitivity in detecting early material motion near the surface. This configuration is inherently sensitive to detecting small isolated particles and permits usage of the detector's full dynamic range. Similar to the S-V configuration, the backscattering configuration shows a jet of ejected material clusters. While not able to detect the shock front in the backscattering mode, this mode has the advantage that it is sensitive to early material response behind the expanding shock front. On the other hand, coherent artifacts like laser speckle can influence the quality of backscattering images which are largely absent in shadowgraphy. This configuration should also be able to detect concentrations of particles smaller than the resolution limit of the system (nano-particles) which will appear as "light scattering clouds."

\subsection{Dual-probe configuration optimized for determining ejecta properties}

The previous two system configurations are able to capture material motion and shock propagation spanning the entire timeline of an exit-surface damage event. These configurations can only capture one transient image per each distinct event. However, information on shock wave and particle trajectory can be more accurately retrieved by imaging the same event at multiple time points, especially when time-dependent motion parameters are involved (i.e., acceleration or deceleration). Therefore, an additional experimental configuration was implemented to better address these issues.

A configuration which adds the capability of capturing two transient images at different delay times per damage event is shown in Fig. 11. The basic system was expanded to include a second 532-nm probe laser whose pulse was delayed with respect to that of the first probe. To differentiate the images from each probe, we utilized a polarization filtering method in which the output polarizations of the two probe beams were set to be orthogonal to each other (i.e., vertical and horizontal). The two beams were then combined into a single beam path using a polarizing beam-splitter. A nonpolarizing cube beam splitter was then used to separate the dual-probe beam into two paths to illuminate the damage site with the same geometry as the basic system (Fig. 6). In this manner, the image formed by the S-V microscope is a composite image of the damage site formed by the two probes delayed in time. A polarizing beam-splitter located behind the microscope's image-forming optics separates the two orthogonal polarization image components and directs them to separate CCD cameras. The time delays between the two probe pulses used for these experiments ranged from 100 to 500 ns.

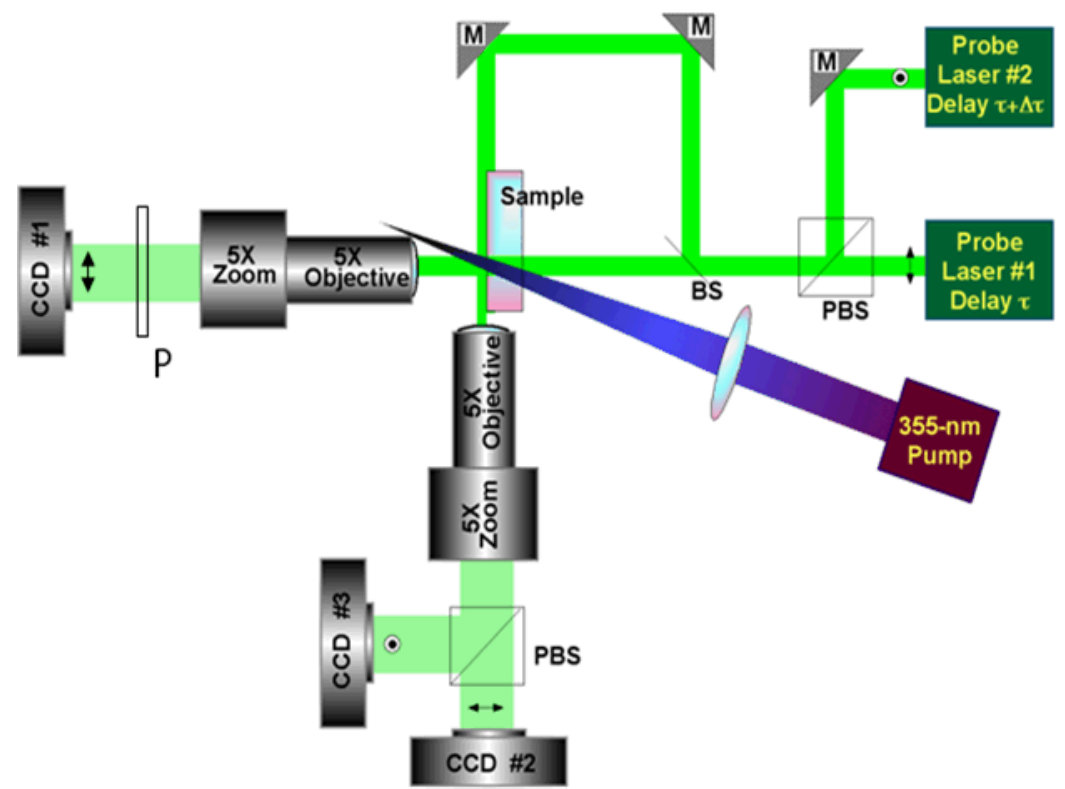

Figure 11. Pump- dual probe imaging configuration for S-V multi-image acquisition of the same damage event at different delay times. 

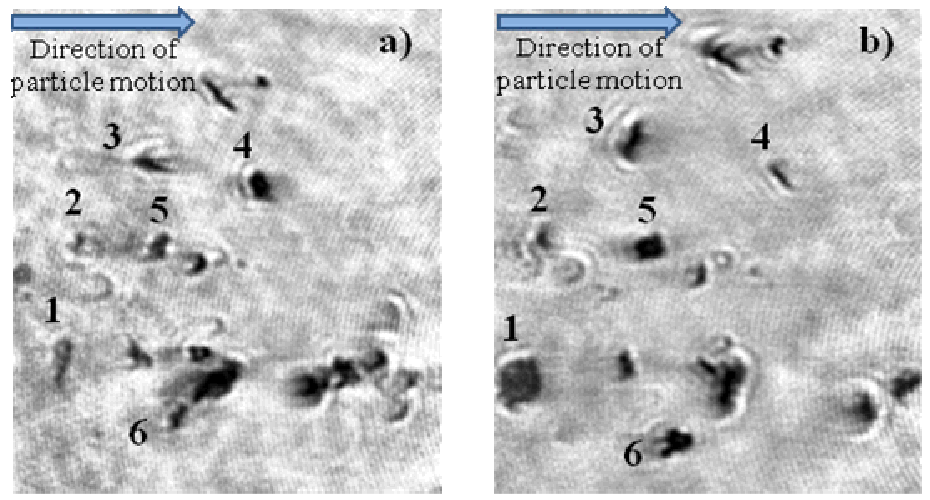

Figure 12. a) Side-view image at pump-probe delay of $2 \mu \mathrm{s}$. b) Image of same event $500 \mathrm{~ns}$ later. Six particles are identified for comparison.

For calibration and image co-registration between the two S-V cameras, probe 2 was turned off and a quarter-waveplate inserted into the probe 1 path to provide illumination of the damage site with circular polarization. This polarization state of the illuminating pulse enabled the acquisition of transient or static images with both CCD cameras by splitting the same image into two polarization components. Subsequently, the position of the cameras was adjusted so that both cameras provided exactly the same magnification, and the location of the surface was determined for each CCD.

Following this calibration process, we were able to accurately quantify the distance traveled by individual particles or shock fronts from their location in the two images captured at different delay times (i.e., dual probe imaging). As a typical example, Fig. 12a shows particle ejecta at $2 \mu$ s delay, while Fig. 12b shows an image of the same event $500 \mathrm{~ns}$ later (at $2.5 \mu \mathrm{sec}$ delay) when the particles have traveled further from the surface. The relative positions of the particles do not change for the most part, so most particles can be easily identified in both images. In addition to translational motion, many particles can be seen to exhibit rotational motion. For example, the particles denoted as 1-6 in Fig. 12 exhibit different projected dimensions at the two time points, thus revealing their 3-dimensional shape.

While only the average dynamic properties of ejected particles are inferable based on single probe imaging, the dualprobe configuration directly measures the average speed of the ejected particles during the time interval between probe 1 and probe 2. This method also provides evidence that particles may have accelerated (for example when crossing the shock front) or decelerated along their trajectory from the surface. In addition to translational kinetic energy, comparison of the two images from this dual probe setup yields information on rotational motion and shape of the ejecta. With this system we are also able to estimate the ejection time of the particles observed in the images captured at different delay times. Preliminary results indicate that the ejection time can be as long as $20 \mu$ s after the arrival of the pump pulse.

\section{DISCUSSION}

The material response to laser damage is multi-faceted, consisting of generation of shock waves, transient material absorption and the generation of extreme temperatures and pressures over a microscopic volume. In the case of bulk damage, transient absorption of the cracks terminated at about $300 \mathrm{~ns}$ while that of the core persisted until $150 \mu \mathrm{s}$. In addition, we observed transient scattering of the core region at delays on the order of tens of milliseconds. It has been postulated that the material in the core region exists transiently in the liquid or vapor phase at an elevated temperature $[12,13]$. However, this phenomenon was previously believed to only last on the order of picoseconds, whereas our results suggest that this process is much more long-lived. Regarding kinetic parameters, we estimated the speed of the initial shock wave to be near the speed of sound in bulk fused silica (longitudinal mode, $5.97 \mathrm{~km} / \mathrm{s}$ ). In addition, we estimated the speed of the secondary shock front to be near the speed of the transverse mode of sound $(3.76 \mathrm{~km} / \mathrm{s})$ in the bulk.

In the case of surface damage, we observed material motion towards the end of the laser pulse. The material swells until about $25 \mathrm{~ns}$, after which the first particles are ejected (with diameters from 1-4 $\mu \mathrm{m}$ and speeds up to $\sim 3 \mathrm{~km} / \mathrm{s}$ ), leading to crater formation. At time delay times near $\sim 10 \mu \mathrm{s}$, the average diameter of ejected particles is larger $(>30 \mu \mathrm{m})$ while the average speed is slower $(<200 \mathrm{~m} / \mathrm{s})$. Fewer, irregularly shaped particles are ejected at even later delays. 


\section{CONCLUSIONS}

In this work, we have presented a versatile time-resolved microscope system to obtain information on the dynamic behavior of the material during initiation through the entire timeline of damage on the surface or in the bulk of fused silica. Various configurations of a single system are described such as simultaneous side-view and transmission-view imaging of distinct events, single and dual probe imaging of the same event at multiple time points, backscattering imaging to visualize the onset of material motion at early delays. Each configuration is designed to best address different aspects of the damage timeline.

\section{ACKNOWLEDGEMENTS}

This work was performed under the auspices of the U.S. Department of Energy by Lawrence Livermore National Laboratory under Contract DE-AC52-07NA27344.

\section{REFERENCES}

[1] Demos, S. G., Staggs, M., Minoshima, K., and Fujimoto, J., "Characterization of laser induced damage sites in optical components" Opt. Express 10(25), 1444-1450 (2002).

[2] Kucheyev, S. O. and Demos, S. G., "Optical defects produced in fused silica during laser-induced breakdown," Appl. Phys. Lett. 82(19), 3230-3232 (2003).

[3] Negres, R. A., Kucheyev, S. O., DeMange, P., Bostedt, C., van Buuren, T., Nelson, A. J., and Demos, S. G., "Decomposition of $\mathrm{KH}_{2} \mathrm{PO}_{4}$ crystals during laser-induced breakdown," Appl. Phys. Let. 86(17), 171107 (2005).

[4] Carr C. W., Feit, M. D., Johnson, M. A., and Rubenchik, A. M., "Complex morphology of laser-induced bulk damage in $\mathrm{K}_{2} \mathrm{H}_{(2-\mathrm{x})} \mathrm{D}_{\mathrm{x}} \mathrm{PO}_{4}$ crystals," Appl. Phys. Lett. 89(13), 131901 (2006).

[5] Wong, J., Ferriera, J. L., Lindsey, E. F., Haupt, D. L., Hutcheon, I. D., and Kinney, J. H., "Morphology and microstructure in fused silica induced by high fluence ultraviolet $3 \omega(355 \mathrm{~nm})$ laser pulses," J. Non-Cryst. Solids 352(3), 255-272 (2006).

[6] Norman, T.J., Zaug, J. M., and Carr, C. W., "High-pressure decomposition of DKDP," Chem. Mater. 18(13), 30743077 (2006).

[7] Negres, R. A., Burke, M. W., DeMange, P. P., Sutton, S. B., Feit, M. D., and Demos, S. G., "Evaluation of UV absorption coefficient in laser-modified fused silica," Appl. Phys. Let. 90(6), 061115 (2007).

[8] Carr, C. W., Radousky, H. B., Rubenchik, A. M., Feit, M .D., and Demos, S. G., "Localized dynamics during laserinduced damage in optical materials," Phys. Rev. Lett. 92(8), 087401 (2004).

[9] Russo, R. E., Mao, X. L., Liu, H. C., Yoo, J. H., and Mao, S. S., "Time-resolved plasma diagnostics and mass removal during single-pulse laser ablation," Appl. Phys. A-Mater. 69, S887-S894 (1999).

[10] Demos, S.G. and Negres, R. A., "Time-resolved imaging of material response during laser-induced bulk damage in $\mathrm{SiO}_{2}$," Proc. SPIE 7132, $71320 \mathrm{Q}$ (2008).

[11] Negres, R. A., Feit, M. D., DeMange, P., Bude, J. D., and Demos, S. G., "Pump and probe damage testing for investigation of transient material modifications associated with laser damage in optical materials," Proc. SPIE 6720, 672019-1-6 (2008).

[12]Feit, M. D. and Rubenchik, A. M., Sell, W. D., and Wegner, P., "Implications of nanoabsorber initiators for damage probability curves, pulselength scaling, and laser conditioning," Proc. SPIE 5273, 74-82 (2004).

[13] Bude, J., Guss, G., Matthews, M., and Spaeth, M. L., "The effect of lattice temperature on surface damage in fused silica optics," Proc. SPIE 6720, 672009 (2007). 